\title{
Theory of Enhanced Interlayer Tunneling in Optically Driven High- $T_{c}$ Superconductors
}

\author{
Jun-ichi Okamoto, ${ }^{1,2, *}$ Andrea Cavalleri, ${ }^{3,4}$ and Ludwig Mathey ${ }^{1,2}$ \\ ${ }^{1}$ Zentrum für Optische Quantentechnologien and Institut für Laserphysik, Universität Hamburg, 22761 Hamburg, Germany \\ ${ }^{2}$ The Hamburg Centre for Ultrafast Imaging, Luruper Chaussee 149, 22761 Hamburg, Germany \\ ${ }^{3}$ Max Planck Institute for the Structure and Dynamics of Matter, 22761 Hamburg, Germany \\ ${ }^{4}$ Department of Physics, Clarendon Laboratory, University of Oxford, Oxford OX1 3PU, United Kingdom
}

(Received 29 June 2016; published 22 November 2016)

\begin{abstract}
Motivated by recent pump-probe experiments indicating enhanced coherent $c$-axis transport in underdoped YBCO, we study Josephson junctions periodically driven by optical pulses. We propose a mechanism for this observation by demonstrating that a parametrically driven Josephson junction shows an enhanced imaginary part of the low-frequency conductivity when the driving frequency is above the plasma frequency, implying an effectively enhanced Josephson coupling. We generalize this analysis to a bilayer system of Josephson junctions modeling YBCO. Again, the Josephson coupling is enhanced when the pump frequency is blue detuned to either of the two plasma frequencies of the material. We show that the emergent driven state is a genuine, nonequilibrium superconducting state, in which equilibrium relations between the Josephson coupling, current fluctuations, and the critical current no longer hold.
\end{abstract}

DOI: 10.1103/PhysRevLett.117.227001

Recent pump-probe experiments on high-temperature superconductors such as YBCO revealed transiently enhanced superconducting-like states both below and above their critical temperatures $T_{c}$ [1-4]. The origin of these transient superconducting states has not been identified yet. Several ideas have been proposed: nonlinear phononic effects [5], parametric cooling [6,7], competing orders [8,9], and redistribution of phase fluctuations [10]. In this Letter, we model the layered structure of $\mathrm{YBCO}$ as a Josephson junction chain, and demonstrate that below the critical temperature the parametric driving of these junctions enhances the Josephson coupling in the steady state [11]. We extract this quantity from the $1 / \omega$ divergence of the imaginary part of the conductivity $\sigma(\omega)$ as the frequency $\omega$ approaches zero.

So far, most of the theoretical studies have considered quantities such as the power spectrum of currents [10], or the dc current response [6]. While these quantities give important insight into the system, here we discuss the optical conductivity itself by including the probing field in our calculation to obtain the actual nonequilibrium response. The Kubo formula or other equilibrium methods are not used. This achievement is crucially important because the conclusions of Refs. [1-4] are based precisely on this quantity. We note that while experiments indicate stiffening of the superfluid density both below and above $T_{c}$, here we focus on cases below $T_{c}$, in which condensed Cooper pairs are safely assumed. We will discuss cases with fluctuating Cooper pairs just above $T_{c}$ (below the pseudogap temperature) elsewhere. Complete understanding how the interlayer coherence is dynamically enhanced may provide new theoretical and experimental means to investigate fluctuating orders above $T_{c}$.
In this Letter, we first study a single Josephson junction. We show that a driving frequency just above the plasma frequency leads to strong enhancement of the Josephson coupling, derived from the low-frequency conductivity. This arises because the nonlinear driving term couples the probe pulse to the plasma frequency. This parametric mechanism can also be considered as a Fano-Feshbach resonance [12]. Then we turn to a bilayer system of Josephson junctions (Fig. 1), which has been used to explain the optical properties of YBCO [13-15]. We employ both an analytical approach and Langevin
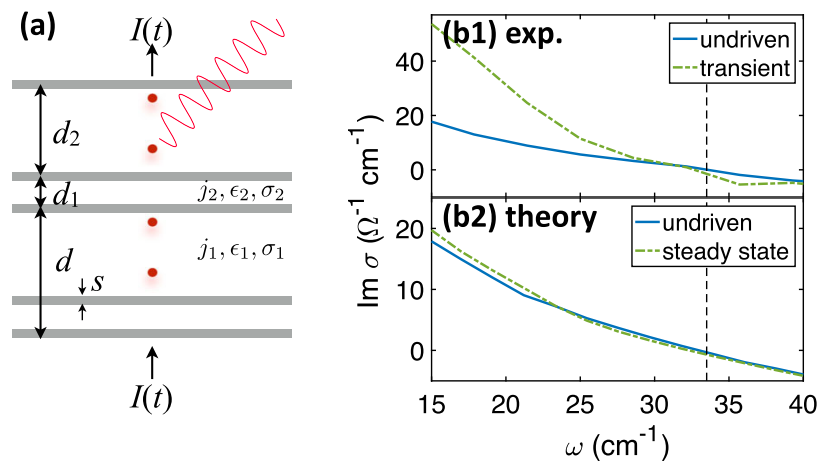

FIG. 1. (a) Schematic depiction of YBCO. Superconducting $\mathrm{CuO}_{2}$ layers (grey) form series of bilayer Josephson junctions. THz pulses (wavy lines) excite apical oxigen atoms (circles) that induce oscillations of $j_{1}$ and $j_{2}$. (b) Comparison of the calculated imaginary part of the conductivity with experiments in Ref. [2] at $10 \mathrm{~K}$. (The lower plasma frequency is $\sim 33 \mathrm{~cm}^{-1}$.) The optimal driven conditions near the higher plasma frequency are used for the calculations. (Theoretical curves are rescaled so that undriven curves from the experiment and theory coincide.) 
simulations to calculate the conductivities. We find that, when the driving frequency is just above the low and high plasma frequencies, the Josephson coupling increases compared to the undriven cases by $20 \%$ and $8 \%$, respectively, in the steady state (Fig. 1). We propose ways to further increase the enhancement; near the low plasma frequency, increasing capacitive couplings of both junctions is ideal, while near the high plasma frequency, increasing (decreasing) the coupling of weak (strong) junctions is preferable. Finally, we demonstrate the counterintuitive properties of this nonequilibrium superconductor. We discuss a scenario where both current fluctuations and the Josephson coupling are enhanced, which would be unexpected in equilibrium. We explain this phenomena by the redistribution of current fluctuations in frequency space.

We start with a single parametrically driven junction [16-21], with a bare Josephson coupling $J_{0}$, a thickness $d$, and a dielectric constant $\epsilon$. It has a characteristic plasma frequency $\omega_{\mathrm{Jp}}=\sqrt{4 \pi e^{*} d J_{0} / \hbar \epsilon}$. The phase $\varphi$ of the junction obeys

$$
\ddot{\varphi}+\gamma \dot{\varphi}+\omega_{\mathrm{Jp}}^{2}\left[1+A \cos \left(\omega_{\mathrm{pump}} t\right)\right] \sin \varphi=\tilde{I},
$$

where $\gamma$ is a damping coefficient, $\tilde{I} \equiv \omega_{\mathrm{Jp}}^{2} I / J_{0}$, and $I$ is an external current. We consider a periodic modulation of $J_{0}$ with an amplitude $A(0<A<1)$ at a frequency $\omega_{\text {pump }}$ as $J_{0} \rightarrow J_{0}\left[1+A \cos \left(\omega_{\text {pump }} t\right)\right]$. This induces parametric driving. $\tilde{I}$ is independent of $A$ since $\omega_{\mathrm{Jp}}^{2} \propto J_{0}$. Linearizing and Fourier transforming the equation, we obtain

$$
\begin{aligned}
& \left(\frac{-\omega^{2}-i \gamma \omega}{\omega_{\mathrm{Jp}}^{2}}+1\right) \varphi(\omega) \\
& \quad=-\frac{A\left[\varphi\left(\omega+\omega_{\text {pump }}\right)+\varphi\left(\omega-\omega_{\text {pump }}\right)\right]}{2}+\tilde{I}(\omega) .
\end{aligned}
$$

To calculate the conductivity, we assume that the probing current is monochromatic $\tilde{I}(\omega)=\tilde{I}_{\text {probe }} \delta\left(\omega-\omega_{\text {probe }}\right)$. We need to solve a discrete set of equations for $\varphi_{n} \equiv \varphi\left(\omega_{\text {probe }}+n \omega_{\text {pump }}\right), n \in \mathbb{Z}$, to determine $\varphi\left(\omega_{\text {probe }}\right)$. Since higher harmonics are of higher order in the driving amplitude, we consider only $\varphi_{ \pm 1}$ and $\varphi_{0}$ [22]. Using the Josephson relation $V=\left(\hbar / e^{*}\right) \dot{\varphi}$, and $\sigma=I d / V$, we find [31]

$$
\begin{aligned}
\sigma\left(\omega_{\text {probe }}\right)= & \frac{\epsilon}{4 \pi i \omega_{\text {probe }}}\left[\frac { A ^ { 2 } \omega _ { \mathrm { Jp } } ^ { 4 } } { 4 } \left(\frac{1}{\omega_{\mathrm{Jp}}^{2}-\left(\omega_{\text {pump }}-\omega_{\text {probe }}\right)\left(-i \gamma+\omega_{\text {pump }}-\omega_{\text {probe }}\right)}\right.\right. \\
& \left.\left.+\frac{1}{\omega_{\mathrm{Jp}}^{2}-\left(\omega_{\text {pump }}+\omega_{\text {probe }}\right)\left(i \gamma+\omega_{\text {pump }}+\omega_{\text {probe }}\right)}\right)+\omega_{\text {probe }}^{2}+i \gamma \omega_{\text {probe }}-\omega_{\mathrm{Jp}}^{2}\right] .
\end{aligned}
$$

In the London and Ginzburg-Landau picture [32],

$$
\operatorname{Im}\left[\sigma_{\text {London }}(\omega)\right]=\frac{n_{S} e^{* 2}}{m \omega}=\frac{c^{2}}{4 \pi \lambda_{L}^{2} \omega}=\frac{J_{0} e^{*} d}{\hbar \omega},
$$

where $n_{S}$ is the superfluid density, $m$ the mass of a Cooper pair, $\lambda_{L}$ the London penetration depth, and $J_{0}$ the bare Josephson coupling. Thus we define an effective Josephson coupling $J_{\text {eff }}$ as

$$
\begin{aligned}
J_{\mathrm{eff}} & \equiv \frac{\hbar}{e^{*} d} \operatorname{Im}[\sigma(\omega) \omega]_{\omega=0} \\
& =J_{0}\left(1-\frac{A^{2} \omega_{\mathrm{Jp}}^{2}\left(\omega_{\mathrm{Jp}}^{2}-\omega_{\text {pump }}^{2}\right)}{2\left(\omega_{\mathrm{Jp}}^{2}-\omega_{\mathrm{pump}}^{2}\right)^{2}+2 \gamma^{2} \omega_{\text {pump }}^{2}}\right) .
\end{aligned}
$$

The driving term generates a resonant renormalization of $J_{0}$, which is the key mechanism that we propose here. When the driving frequency is above (below) the plasma frequency, the effective coupling is enhanced (decreased). The maximal value of the correction $J_{\text {eff }} / J_{0}=$ $1+A^{2} \omega_{\mathrm{Jp}}^{2} / 2 \gamma\left(\gamma+2 \omega_{\mathrm{Jp}}\right) \quad$ is achieved at $\omega_{\text {pump }}=$ $\omega_{\mathrm{Jp}} \sqrt{1+\gamma / \omega_{\mathrm{Jp}}}$. We note that Eq. (5) is of the form of a Fano-Feshbach resonance [12]; the plasma mode corresponds to the bound state, and the pump-pulse couples the probing mode to this bound state.

In the Supplemental Material [22], we include the next order of the nonlinearity of $\sin \varphi$ in Eq. (1). We show the result of this extended analysis in Fig. 2 for $\gamma=0.05$. We exclude the regions of dynamical instability determined by Floquet analysis of the linear model [22-24]. As the

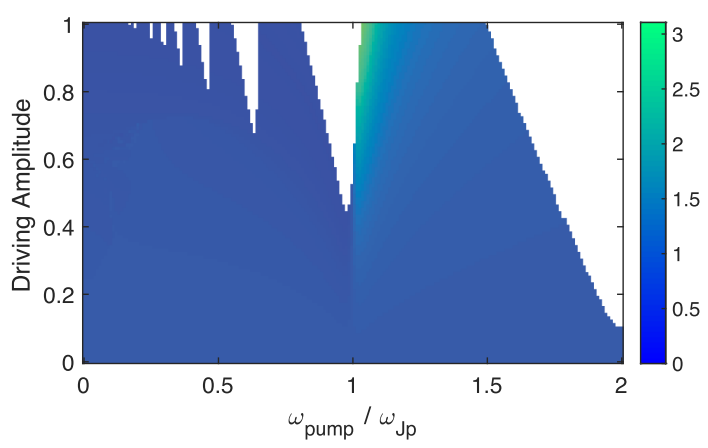

FIG. 2. The normalized effective Josephson coupling $J_{\text {eff }} / J_{0}$. We note that a significant increase of the coupling is achieved when the driving frequency is just above the plasma frequency. Dynamically unstable regions are left blank. 
damping $\gamma$ increases, the instability regions diminish while the enhancement of $J_{\text {eff }}$ also decreases; for the optimal gain of $J_{\text {eff }}$, it is desirable to control the damping to balance these two effects.

Next we consider a bilayer system of $2 \mathrm{~N}$ Josephson junctions composed of $2 N+1$ superconducting (SC) layers stacked along the $c$ axis that models the electromagnetic response of YBCO [2,14,15] (Fig. 1). The $m$ th superconducting layer has the excess charge $Q_{m}$ and the phase of the superconducting order parameter $\theta_{m}$. These are conjugate variables, satisfying $\left\{\theta_{l},-(\hbar / 2 e) Q_{m}\right\}=\delta_{l m}$. Between neighboring superconducting layers is an insulating layer, creating the Josephson junctions. There are two types of Josephson junctions in our model, labeled as " 1 " and " 2 " or equivalently "weak" and "strong." These are of thickness $d_{1,2}$, and consist of one unit cell of thickness $d=d_{1}+d_{2}$ (Fig. 1). Each junction is characterized by a high-frequency dielectric constant $\epsilon_{1,2}$ and a Josephson critical current $j_{1,2}$. The average dielectric constant for the unit cell is $\epsilon_{\mathrm{av}}^{-1}=\left(d_{1} \epsilon_{1}^{-1}+d_{2} \epsilon_{2}^{-1}\right) / d$, and the average capacitance is $C_{\mathrm{av}}=W \epsilon_{\mathrm{av}} / 4 \pi d$. $W$ is the area of the layers. In addition to the self-charging energy that arises from the nonzero compressibility of each superconducting layer, there are long-range Coulomb interactions among the excess charges $Q_{m}$ due to lack of screening charges in the insulating layers. The total Hamiltonian is $[14,15]$

$$
\begin{aligned}
H= & -\sum_{m>n} \frac{\left|x_{m}-x_{n}\right|}{2 d C_{\mathrm{av}}} Q_{m} Q_{n}+\sum_{m} \frac{\kappa}{2 C_{\mathrm{av}}} Q_{m}^{2} \\
& -\sum_{m} \frac{\hbar j_{m}^{m+1} W}{e^{*}} \cos \left(\theta_{m+1}-\theta_{m}\right),
\end{aligned}
$$

where $x_{m}$ is the coordinate of the $m$ th SC layer, with $x_{2 m}=m d$, and $x_{2 m+1}=m d+d_{1}$. The first term represents the Coulomb interactions. The second term is the selfcharging energy with a dimensionless compressibility $\kappa=\epsilon_{\mathrm{av}} \mu^{2} / s d$, with $s$ being the thickness of the superconducting layer. $\mu$ is the Thomas-Fermi screening length in the superconducting layers. The last term is the Josephson energy term. $j_{m}^{m+1}=j_{1,2}$ is the Josephson critical current between the $m$ th and the $(m+1)$ th SC layer. The Hamilton equations obtained from Eq. (6) are coupled sine-Gordon equations for $2 \mathrm{~N}$ junctions [22]. Here we show the simplified equations where all the weak/strong junctions are set to be equal, i.e., $\varphi_{1}=\varphi_{3}=\ldots$ and $\varphi_{2}=\varphi_{4}=\ldots$,

$$
\begin{aligned}
& {\left[\begin{array}{c}
\ddot{\varphi}_{1} \\
\ddot{\varphi_{2}}
\end{array}\right]+\gamma\left[\begin{array}{c}
\dot{\varphi}_{1} \\
\dot{\varphi_{2}}
\end{array}\right]-\frac{4 \pi e^{*} \mu^{2} I}{s}\left[\begin{array}{c}
\alpha_{1}^{-1} \\
\alpha_{2}^{-1}
\end{array}\right]} \\
& \quad=\left[\begin{array}{cc}
-\left(1+2 \alpha_{1}\right) \Omega_{1}^{2} & 2 \alpha_{2} \Omega_{2}^{2} \\
2 \alpha_{1} \Omega_{1}^{2} & -\left(1+2 \alpha_{2}\right) \Omega_{2}^{2}
\end{array}\right]\left[\begin{array}{l}
\varphi_{1} \\
\varphi_{2}
\end{array}\right] .
\end{aligned}
$$

$\alpha_{i}$ is the capacitive coupling constant $\alpha_{i}=\epsilon_{i} \mu^{2} / s d_{i}$, and $\Omega_{1,2}$ is the bare plasma frequency of each junction [see the expression above Eq. (1)]. The parametric driving is included by changing the critical currents $j_{i}$ as $j_{1,2} \rightarrow j_{1,2}\left[1 \pm A_{1,2} \cos \left(\omega_{\text {pump }} t\right)\right]$; we assume that the driving is alternating along the junctions. The voltage is related to the phase differences by the generalized Josephson relations [15,33],

$$
\left(\frac{\hbar}{e^{*}}\right)\left[\begin{array}{c}
\dot{\varphi}_{1} \\
\dot{\varphi}_{2}
\end{array}\right]=\left[\begin{array}{cc}
1+2 \alpha_{1} & -2 \alpha_{2} \\
-2 \alpha_{1} & 1+2 \alpha_{2}
\end{array}\right]\left[\begin{array}{l}
V_{1} \\
V_{2}
\end{array}\right] .
$$

For the undriven case at $T=0$, Eqs. (7) and (8) give

$\sigma(\omega)=\frac{\epsilon_{\mathrm{av}}}{4 \pi i} \frac{\left(\omega^{2}+i \gamma \omega-\omega_{\mathrm{Jp} 1}^{2}\right)\left(\omega^{2}+i \gamma \omega-\omega_{\mathrm{Jp} 2}^{2}\right)}{\omega\left(\omega^{2}+i \gamma \omega-\omega_{t}^{2}\right)}$,

where $\omega_{\mathrm{Jp} 1, \mathrm{Jp} 2} \simeq \Omega_{1,2}$ are the longitudinal plasma modes for weak and strong junctions, and $\omega_{t} \simeq \omega_{\mathrm{Jp} 2}$ is the transverse plasma mode $[15,22]$. The overall Josephson coupling at $T=0$, defined as Eq. (5), is $J_{0}=$ $e^{*} \epsilon_{\mathrm{av}} \omega_{\mathrm{Jp} 1}^{2} \omega_{\mathrm{Jp} 2}^{2} /\left(4 \pi \hbar d \omega_{t}^{2}\right)$.

We calculate the effective Josephson coupling for the driven state, similar to the single junction case. Assuming $\omega_{\mathrm{Jp} 1}, \Omega_{1} \ll \omega_{\mathrm{Jp} 2}, \Omega_{2}$ and $A_{i}, \gamma \ll 1$, and with a pump frequency near the lower resonance, $\omega_{\mathrm{pump}} \simeq \omega_{\mathrm{Jp} 1}$, the relative change of the Josephson coupling $\delta J / J_{0}$ is approximately [22]

$$
\delta J / J_{0} \simeq \frac{A_{1}^{2} \omega_{\mathrm{Jp} 1}^{2}}{2\left(\omega_{\mathrm{pump}}^{2}-\omega_{\mathrm{Jp} 1}^{2}\right)}\left(1+2 \alpha_{1}+2 \alpha_{2}\right) .
$$

This indicates that increasing both capacitive couplings further enhances the Josephson coupling. Near the higher resonance $\omega_{\text {pump }} \simeq \omega_{\mathrm{Jp} 2}$, we find

$\delta J / J_{0} \simeq \frac{-2 \alpha_{2}^{2} A_{1}^{2}+\alpha_{1}\left(1+2 \alpha_{2}\right) A_{2}^{2}+4 \alpha_{1} \alpha_{2} A_{1} A_{2}}{2 \alpha_{2}\left(\omega_{\text {pump }}^{2}-\omega_{\mathrm{Jp} 2}^{2}\right)} \Omega_{1}^{2}$.

In the pump-probe experiment on YBCO [2], the driving frequency is above $\omega_{\mathrm{Jp} 2}$. For that frequency regime, we propose to use a material with a smaller $\alpha_{2}$ or a larger $\alpha_{1}$ to exploit the singular behavior of Eq. (11).

Next, we solve the $2 \mathrm{~N}$ coupled sine-Gordon equations with Langevin noise terms numerically to study thermal effects. We focus on the response below the critical temperature since the model assumes condensed Cooper pairs; only the interlayer phase fluctuations are active. We do not address the physics above the critical temperature. For the numerical integration, we employ a Heun scheme with typical time steps of $h=10^{-5}$, with $N=50$ unit cells. The other parameters of the model are $\alpha_{1}=3, \alpha_{2}=1.5$, $\Omega_{1}=1, \Omega_{2}=12.5$, and $\gamma=0.5$. These are chosen to reproduce the ratio $\omega_{\mathrm{Jp} 2} / \omega_{\mathrm{Jp} 1} \sim 15.8$ of YBCO with appropriate $\alpha$ values for this compound of around $\sim 3$ [15]. We have $\omega_{\mathrm{Jp} 1}=1.58$ and $\omega_{\mathrm{Jp} 2}=25.1$. We use a small $\gamma<\Omega_{i}$ 
to simulate the underdamped regime. To calculate the conductivity $\sigma\left(\omega_{\text {probe }}\right)$, we add a monochromatic probing current at $\omega_{\text {probe }}$ with $I=0.1$ [34], and measure the voltage across the junctions. The numerically obtained response functions in equilibrium are in good agreement with the analytical expressions in Eq. (9) [22]. Because of the lowdimensional nature of the system, it does not undergo a true phase transition at nonzero temperature. However, the Josephson coupling decreases rapidly near the crossover temperature $T \sim 1.0$; see Fig. 3(c) [35].

The effective Josephson coupling obtained from the conductivity at $T=0.2$ and 0.6 are plotted in Figs. 3(a) and 3(b) near the low and high plasma frequencies. We choose maximal driving amplitudes that are still inside the dynamically stable regions, $\left(A_{1}, A_{2}\right)=(0.6,0.3)$ and $(1.0,0.3)$, respectively. We also plot the analytical solution, including three harmonics, at $T=0$ for comparison. Similar to the single junction, we observe an enhancement of $J_{\text {eff }}$ just above the two plasma frequencies. The largest relative increases are $20 \%$ and $8 \%$, respectively, for these examples, in the steady state. Near the lower resonance, the deviation from the analytical solution is more significant than for the higher resonance, since the higher harmonics become more important in this case. The analytical expression agrees better with the $T=0.2$ results, and deviates further from the curves for $T=0.6$ due to thermal effects, especially for the lower resonance. The temperature dependence of $J_{\text {eff }}$ at fixed driving frequencies $\omega_{\text {pump }}=2.1$ and 26 is depicted in Fig. 3(c). The enhanced Josephson coupling rapidly decreases as the temperature increases above the lower resonance at $\omega_{\text {pump }}=2.1$. Near the higher
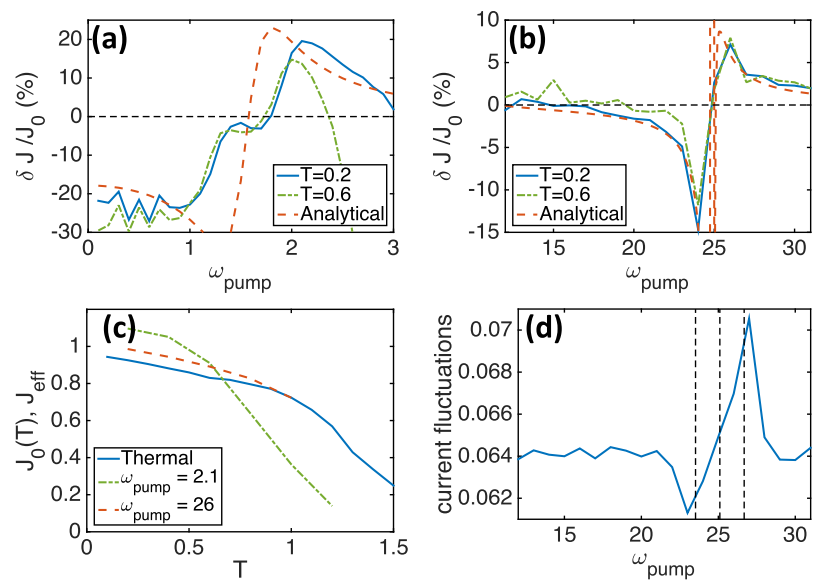

FIG. 3. (a) The relative change of the Josephson coupling $\delta J / J_{0}(\%)$ near $\omega_{\text {pump }} \simeq \omega_{\mathrm{Jp} 1}$ at $A_{1}=0.6$ and $A_{2}=0.3$. (b) The relative change of the Josephson coupling $\delta J / J_{0}(\%)$ near $\omega_{\text {pump }} \simeq \omega_{\mathrm{Jp} 2}$ at $A_{1}=1.0$ and $A_{2}=0.3$. (c) The temperature dependence of $J_{0}$ and $J_{\text {eff }}$ [normalized by $J_{0}(0)$ ] at fixed driving frequencies, $\omega_{\text {pump }}=2.1$ and 26 . (d) The current fluctuations at the weak junctions, $\overline{\left\langle\sin ^{2} \varphi_{i}\right\rangle_{i \in \text { weak }}}$, near $\omega_{\text {pump }} \simeq \omega_{\mathrm{Jp} 2}$. The dashed lines are at $\omega_{\mathrm{Jp} 2}, \omega_{\mathrm{Jp} 2} \pm \omega_{\mathrm{Jp} 1}$. resonance, the enhancement continues up to the crossover temperature of $T_{c} \simeq 1.0$.

Since the weak junctions dominate the low frequency behavior of the system, we plot the current fluctuations in the weak junctions, $\overline{\left\langle\sin \varphi_{i}^{2}\right\rangle_{i \in \text { weak }}}$, in Fig. 3(d). We denote temporal averages by bars and spatial averages by angle brackets. The pump frequency is near $\omega_{\text {pump }} \sim \omega_{\mathrm{Jp} 2}$, close to the experimental conditions of Ref. [2]. Near $\omega_{\text {pump }} \sim \omega_{\mathrm{Jp} 2} \pm \omega_{\mathrm{Jp} 1}$, the current fluctuations increase (decrease) due to parametric heating (cooling) as has been observed in Ref. [6]. The enhanced Josephson coupling near $\omega_{\text {pump }} \simeq 26$ occurs when current fluctuations increase due to parametric heating. For an equilibrium system, this would be unexpected, because larger fluctuations are achieved for higher temperatures and lead to a smaller superfluid density and Josephson coupling. In equilibrium, the thermal fluctuations for all frequencies are controlled by a single parameter, the temperature. Here, however, we have created a genuine nonequilibrium state. To demonstrate this, we generate the power spectrum of the weak junction currents, which is the Fourier transform of the temporal currentcurrent correlation function $\overline{I_{\mathrm{w}}(t) I_{\mathrm{w}}(0)}$ where $I_{\mathrm{w}} \equiv\left\langle\sin \varphi_{i}\right\rangle_{i \in \text { weak }}$. This spectrum is shown in Fig. 4(a) near the parametric heating regime $\omega_{\text {pump }}=26$. The low frequency part of the power spectrum is related to the conductivity [22], while its sum over all frequencies is equal to the variance $\overline{\left\langle\sin \varphi_{i}^{2}\right\rangle_{i \in \text { weak }}}$. We observe that the low frequency part $\left(\omega<\omega_{\mathrm{Jp} 1}\right)$ has reduced fluctuations, as if the temperature were reduced. This leads to the enhancement of conductivity. However, the total area of the spectrum is increased because the high frequency fluctuations are increased, as if their temperature was increased [36]. We note that the dc current response, which serves as another estimate of Josephson energy [6], is determined by the total power spectrum, and therefore may be different from $J_{\text {eff }}$, which is controlled by the low frequency part. Indeed, we find that the parametric heating (cooling) gives more (fewer) phase slips resulting in larger (smaller) voltages near the critical current, as shown in the dc current-voltage curves in Fig. 4(b). This agrees with the results of Ref. [6].

In conclusion, we have demonstrated a mechanism for light-enhanced superconductivity in which the probe pulse is coupled to a plasma mode by parametric driving. We have first showed this phenomenon for a single, parametrically
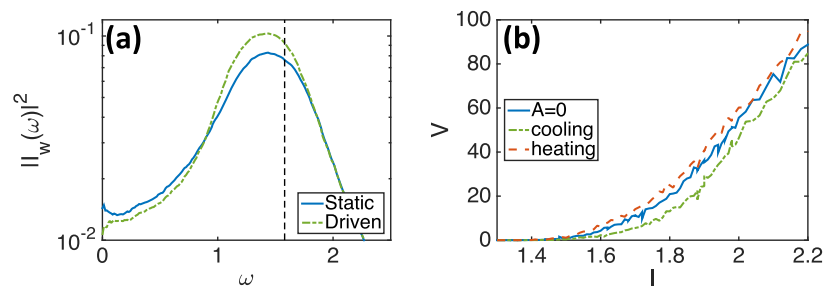

FIG. 4. (a) Power spectrum of the weak junction current at $T=$ 0.6 around $\omega_{\mathrm{Jp} 1}$; (b) dc current-voltage characteristics. 
driven Josephson junction. We have then expanded this analysis to a bilayer system of Josephson junctions which represents the phase and charge dynamics along the $c$ axis of YBCO. Both of these models indeed show an enhanced Josephson coupling when the pump frequency is above the plasma frequencies. We find that smaller damping increases the enhancement, while too little damping induces a parametric instability. We have also proposed material properties that are beneficial for an enhanced Josephson coupling. Finally, we have demonstrated that the resulting driven state is a genuine nonequilibrium state in which enhanced Josephson coupling and increased current fluctuations coexist. We emphasize that the conceptual features of our mechanism provide guidance for a wide range of driven solid state or other many-body systems, in particular for the dynamical control of their low-frequency response functions.

This work was supported by the Deutsche Forschungsgemeinschaft through the SFB 925 and the Hamburg Centre for Ultrafast Imaging, and from the Landesexzellenzinitiative Hamburg, supported by JoachimHerzStiftung. We thank W. Hu, Y. Laplace, D. Nicoletti, C. Zerbe, R. Höppner, T. Rexin, and B. Zhu for helpful discussions.

*ojunichi@physnet.uni-hamburg.de

[1] D. Fausti, R. I. Tobey, N. Dean, S. Kaiser, A. Dienst, M. C. Hoffmann, S. Pyon, T. Takayama, H. Takagi, and A. Cavalleri, Light-induced superconductivity in a stripe-ordered cuprate, Science 331, 189 (2011).

[2] W. Hu, S. Kaiser, D. Nicoletti, C. R. Hunt, I. Gierz, M. C. Hoffmann, M. Le Tacon, T. Loew, B. Keimer, and A. Cavalleri, Optically enhanced coherent transport in $\mathrm{YBa}_{2} \mathrm{Cu}_{3} \mathrm{O}_{6.5}$ by ultrafast redistribution of interlayer coupling, Nat. Mater. 13, 705 (2014).

[3] S. Kaiser, C. R. Hunt, D. Nicoletti, W. Hu, I. Gierz, H. Y. Liu, M. Le Tacon, T. Loew, D. Haug, B. Keimer, and A. Cavalleri, Optically induced coherent transport far above $T_{c}$ in underdoped $\mathrm{YBa}_{2} \mathrm{Cu}_{3} \mathrm{O}_{6+\delta}$, Phys. Rev. B 89, 184516 (2014).

[4] M. Först, A. Frano, S. Kaiser, R. Mankowsky, C. R. Hunt, J. J. Turner, G. L. Dakovski, M. P. Minitti, J. Robinson, T. Loew, M. Le Tacon, B. Keimer, J. P. Hill, A. Cavalleri, and S. S. Dhesi, Femtosecond x rays link melting of chargedensity wave correlations and light-enhanced coherent transport in $\mathrm{YBa}_{2} \mathrm{Cu}_{3} \mathrm{O}_{6.6}$, Phys. Rev. B 90, 184514 (2014).

[5] R. Mankowsky, A. Subedi, M. Först, S. O. Mariager, M. Chollet, H. T. Lemke, J. S. Robinson, J. M. Glownia, M. P. Minitti, A. Frano, M. Fechner, N. A. Spaldin, T. Loew, B. Keimer, A. Georges, and A. Cavalleri, Nonlinear lattice dynamics as a basis for enhanced superconductivity in $\mathrm{YBa}_{2} \mathrm{Cu}_{3} \mathrm{O}_{6.5}$, Nature (London) 516, 71 (2014).

[6] S. J. Denny, S. R. Clark, Y. Laplace, A. Cavalleri, and D. Jaksch, Proposed Parametric Cooling of Bilayer Cuprate Superconductors by Terahertz Excitation, Phys. Rev. Lett. 114, 137001 (2015).
[7] M. Knap, M. Babadi, G. Refael, I. Martin, and E. Demler, Dynamical Cooper pairing in nonequilibrium electronphonon systems, arXiv:1511.07874.

[8] Z. M. Raines, V. Stanev, and V. M. Galitski, Enhancement of superconductivity via periodic modulation in a three-dimensional model of cuprates, Phys. Rev. B 91, 184506 (2015).

[9] A. A. Patel and A. Eberlein, Light-induced enhancement of superconductivity via melting of competing bond-density wave order in underdoped cuprates, Phys. Rev. B 93, 195139 (2016).

[10] R. Höppner, B. Zhu, T. Rexin, A. Cavalleri, and L. Mathey, Redistribution of phase fluctuations in a periodically driven cuprate superconductor, Phys. Rev. B 91, 104507 (2015).

[11] We assume that the pump pulse primarily excites the apical oxygen and its IR phonons, while Raman phonons may couple to the IR mode.

[12] C. Chin, R. Grimm, P. Julienne, and E. Tiesinga, Feshbach resonances in ultracold gases, Rev. Mod. Phys. 82, 1225 (2010).

[13] D. van der Marel and A. A. Tsvetkov, Transverse optical plasmons in layered superconductors, Czech. J. Phys. 46, 3165 (1996).

[14] D. van der Marel and A. A. Tsvetkov, Transverse-optical Josephson plasmons: Equations of motion, Phys. Rev. B 64, 024530 (2001).

[15] T. Koyama, Josephson plasma resonances and optical properties in high- $T_{c}$ superconductors with alternating junction parameters, J. Phys. Soc. Jpn. 71, 2986 (2002).

[16] P. Jung, Periodically driven stochastic systems, Phys. Rep. 234, 175 (1993).

[17] C. Zerbe, P. Jung, and P. Hänggi, Brownian parametric oscillators, Phys. Rev. E 49, 3626 (1994).

[18] N. W. McLachlan, Theory and Application of Mathieu Functions (Clarendon, New York, 1951).

[19] A. H. Nayfeh and D. T. Mook, Nonlinear Oscillations (Wiley, New York, 2008).

[20] R. Citro, E. G. Dalla Torre, L. D’Alessio, A. Polkovnikov, M. Babadi, T. Oka, and E. Demler, Dynamical stability of a many-body Kapitza pendulum, Ann. Phys. (Amsterdam) 360, 694 (2015).

[21] B. Zhu, T. Rexin, and L. Mathey, Magnus expansion approach to parametric oscillator systems in a thermal bath, Z. Naturforsch. A 71, 921 (2016).

[22] See Supplemental Material at http://link.aps.org/ supplemental/10.1103/PhysRevLett.117.227001, which includes Refs. [13,14,16,23-30], for details of calculations and models.

[23] A. Coddington and R. Carlson, Linear Ordinary Differential Equations (Society for Industrial and Applied Mathematics, Philadelphia, 1997).

[24] L. Cesari, Asymptotic Behavior and Stability Problems in Ordinary Differential Equations, Ergebnisse der Mathematik und ihrer Grenzgebiete. 2. Folge (Springer, Berlin, Heidelberg, 2012).

[25] S. Rajasekaran, E. Casandruc, Y. Laplace, D. Nicoletti, G. D. Gu, and S.R. Clark, Parametric amplification of a superconducting plasma wave, Nat. Phys. 12, 1012 (2016).

[26] M. Machida and S. Sakai, Unified theory for magnetic and electric field coupling in multistacked Josephson junctions, Phys. Rev. B 70, 144520 (2004). 
[27] T. Koyama, Quantum charge dynamics in an array of intrinsic Josephson junctions, J. Phys. Soc. Jpn. 70, 2114 (2001).

[28] R. M. Mazo, On the Brownian motion of a frequencymodulated oscillator, J. Stat. Phys. 24, 39 (1981).

[29] H. Risken and T. Frank, The Fokker-Planck Equation: Methods of Solution and Applications, Springer Series in Synergetics (Springer, Berlin, Heidelberg, 1996).

[30] E. Šimánek, Inhomogeneous Superconductors: Granular and Quantum Effects, International series of monographs on physics (Oxford University Press, Oxford, England, 1994).

[31] The loss function can be also measured similarly by the probing current, and is defined as $L\left(\omega_{\text {probe }}\right) \equiv$ $-\operatorname{Im}\left[\omega_{\text {probe }} / 4 \pi i \sigma\left(\omega_{\text {probe }}\right)\right]$. We confirmed a negative value of loss function near $\omega_{\text {pump }} \simeq 2 \omega_{\text {Jp }}$ as was observed in a
$\mathrm{LaBaCuO}$ material in Ref. [25]. For the details see Supplemental Material [22].

[32] M. Tinkham, Introduction to Superconductivity: Second Edition, Dover Books on Physics (Dover Publications, New York, 2004).

[33] T. Koyama and M. Tachiki, $I-V$ characteristics of Josephson-coupled layered superconductors with longitudinal plasma excitations, Phys. Rev. B 54, 16183 (1996).

[34] We checked that the results are independent of the magnitude of $I$ for $I \leq 0.2$.

[35] The temperature is normalized by $\hbar j_{1} W / \alpha_{1} \Omega_{1}^{2} e^{*}$.

[36] Similar results can be obtained with the linearized model of Eq. (7) based on a Green's functions method. See Supplemental Material [22]. 